\title{
Expansão dos cursos de Graduação em Enfermagem e mercado de trabalho: reproduzindo desigualdades?
}

\author{
Expansion of Undergraduate Nursing and the labor market: reproducing inequalities? \\ Expansión de los cursos de Grado en Enfermería y el mercado laboral: ¿se reproducen las desigualdades?
}

\author{
Kênia Lara Silva', Roseni Rosângela de Sena", Tatiana Silva Tavares"', Lucas Wan der Maas'v \\ ' Universidade Federal de Minas Gerais, Escola de Enfermagem, Núcleo de Estudos e Pesquisas sobre \\ Ensino e Prática de Enfermagem. Belo Horizonte-MG, Brasil. \\ "Universidade Federal de Minas Gerais, Escola de Enfermagem. Belo Horizonte-MG, Brasil. \\ II' Universidade Federal de Minas Gerais, Escola de Enfermagem, Programa de Pós-Graduação (Mestranda), Núcleo de \\ Estudos e Pesquisas sobre Ensino e Prática de Enfermagem. Belo Horizonte-MG, Brasil. \\ Iv Universidade Federal de Minas Gerais, Faculdade de Medicina, Observatório de Recursos Humanos em Saúde, Estação \\ de Pesquisa de Sinais de Mercado, Núcleo de Educação em Saúde Coletiva. Belo Horizonte-MG, Brasil.
}

\section{Submissão: 22-03-2012 Aprovação: 27-07-2012}

\section{RESUMO}

O objetivo do estudo foi analisar a relação entre a expansão dos cursos de graduação de enfermagem e o mercado de trabalho dos enfermeiros em Minas Gerais. Estudo descritivo-exploratório de abordagem quantitativa, que utilizou fontes de dados secundários sobre os cursos de Graduação em Enfermagem, número de profissionais ocupados, vínculos em estabelecimentos de saúde e empregos formais de enfermeiros no Estado. A evolução dos cursos de Graduação em Enfermagem revela sinais de declínio na procura e oferta nos anos recentes, que pode estar relacionado ao mercado de trabalho do enfermeiro. Este, por sua vez, é marcado por contradições expressas pelo quantitativo excedente de profissionais, em especial nas regiões menos desenvolvidas do Estado, bem como pela baixa proporção de enfermeiros para atender as necessidades de saúde da população. Visualiza-se que estes aspectos reproduzem desigualdades e carecem discussões quanto à regulação da formação e à criação de novos postos de trabalho. Descritores: Enfermagem; Formação; Mercado de trabalho.

ABSTRACT
This study aimed to analyze the relationship between the increase in the number of degree courses in nursing and the nursing job market. It is a descriptive exploratory study with a quantitative approach, which used data on Undergraduate Nursing courses, supply of nurses, connection with health facilities, and formal jobs in nursing in the state of Minas Gerais. The evolution of Undergraduate Nursing courses reveals a supply and demand decline in recent years. Such context is determined by the nurse's labor market being influenced by the contradiction of a professional quantitative surplus, particularly in the state's less developed areas, as opposed to a low percentage of nurses to care for the population's health. These characteristics of the nursing labor market reproduce inequalities furthermore aspects such as the regulation of nursing education and the creation of new jobs need to be discussed further.

Key words: Nursing; Education; Labor Market.

\section{RESUMEN}

El objeto del presente estudio fue analizar la relación entre la expansión de los cursos de grado y el mercado laboral de los enfermeros. Estudio exploratorio descriptivo con enfoque cualitativo cuya fuente de información fueron datos sobre los cursos de grado en enfermería, cantidad de enfermeros, vínculos en establecimientos de salud y puestos de trabajo formales de enfermeros del Estado de Minas Gerais. La evolución de los cursos indica disminución de oferta y demanda en los últimos años, contexto determinado por el mercado laboral del enfermero, infuenciado por la contradicción entre el número excedente de profesionales, especialmente en las regiones menos desarrolladas del Estado, y su baja proporción para atender a la población Se percibe que estos aspectos reproducen desigualdades y que debe debatirse más tanto la regulación de la formación del curso como la creación de nuevos puestos de trabajo.

Palabras clave: Enfermería; Formación; Mercado laboral. 


\section{INTRODUÇÃO}

As transformações no quadro político, econômico e social no país têm sido fatores determinantes das características do ensino de enfermagem. Da mesma forma, as necessidades do mercado de trabalho têm influenciado a criação de escolas e a orientação da formação do enfermeiro no Brasil ${ }^{(1)}$.

Dados sobre o número de matrículas em cursos de Graduação em Enfermagem no Brasil indicam que o número quase dobrou em quatro anos, considerando dados de 2004 e 2008: passou de 120.851 para $224.330^{(2)}$. Temos que considerar que a expansão dos cursos representa democratização do acesso ao ensino superior e maior disponibilidade de profissionais no mercado. Contudo, há que se indagar sobre a oferta de postos de trabalho para o enfermeiro, bem como a relação entre a qualidade e a quantidade dos profissionais requeridos para a consolidação do Sistema Único de Saúde.

Neste sentido, apesar do expressivo quantitativo da força de trabalho de Enfermagem, em número absoluto, e da ampliação do número de escolas nas últimas décadas, no país, a concentração e a disponibilidade de profissionais de enfermagem por habitante são bastante desiguais e insuficientes em algumas regiões. Isso porque as escolas de enfermagem concentram-se nas regiões de maior densidade populacional e de concentração de renda do País, acompanhando a distribuição do produto interno bruto, reafirmando as desigualdades regionais ${ }^{(3)}$.

É importante destacar que o mercado de trabalho não é algo estático, pois sofre influências conjunturais ${ }^{(4-5)}$. O mercado de trabalho em saúde enfrenta problemas particulares e desequilíbrios em sua força de trabalho que afetam de forma diferente as regiões de um mesmo país. Esses problemas são relacionados, nas Américas, à quantidade, adequação e capacidade do pessoal de acordo com as necessidades do país; à escassez e aos deslocamentos dos trabalhadores de saúde que superam, em muitos casos, a capacidade dos países de garantir níveis mínimos de atenção, muitas vezes nas zonas ou atividades que mais necessitam. Ressalta-se, ainda, a dificuldade dos países em definir de forma satisfatória um quadro da situação de recursos humanos e uma política de longo prazo para enfrentar os problemas identificados; e a inadequação dos sistemas de gestão do trabalho que nem sempre permitem aos trabalhadores prestar os melhores serviços de forma produtiva e com qualidade, mesmo nos casos em que os países tenham quantidades suficientes de trabalhadores de saúde ${ }^{(6)}$.

No setor saúde, o mercado de trabalho apresentou crescimento a partir da década de 1990, tanto no setor público quanto no privado. Entre os fatores que contribuíram para este crescimento podemos citar a implementação do SUS, que demandou o aparecimento de novas ocupações e a criação de postos de trabalho para fazer frente ao modelo assistencial em implantação ${ }^{(4)}$. A implantação de novos modelos assistenciais, como a Estratégia Saúde da Família (ESF) representou uma importante expansão do mercado de trabalho e, consequentemente, um desafio para a área de recursos humanos, tanto no plano quantitativo e distributivo dos profissionais de saúde, quanto nas possibilidades de qualificação do profissional já inserido no mercado de trabalho ${ }^{(2)}$.

Destacamos neste estudo, o Estado de Minas Gerais pela sua ampla extensão territorial e pelas disparidades regionais. Há diferenciais populacionais e econômicos entre os seus 853 municípios e a educação é um condicionante da desigualdade regional no Estado ${ }^{(7)}$. Considerando o exposto, o estudo teve como objetivo analisar a expansão dos cursos de Graduação de Enfermagem no Estado de Minas Gerais e estabelecer relações com o mercado de trabalho dos enfermeiros.

\section{ASPECTOS METODOLÓGICOS}

Trata-se de um estudo descritivo-exploratório de abordagem quantitativa, conduzido a partir de fontes de dados secundários na área da formação e do mercado de trabalho. $\mathrm{Na}$ área da formação foram utilizados os dados dos cursos de enfermagem de Minas Gerais disponíveis no Censo da Educação Superior do Instituto Nacional de Estudos e Pesquisas Educacionais Anísio Teixeira, do Ministério da Educação e Cultura (INEP/MEC). Foram extraídas as informações de número de cursos, vagas, ingressos e egressos. Na área do mercado nacional de trabalho de enfermeiros, utilizaram-se os dados sobre profissionais ocupados e postos de trabalho de enfermeiros do Cadastro Nacional de Estabelecimentos de Saúde (CNES), do Ministério da Saúde, e da Relação Anual de Informações Sociais (RAIS), do Ministério do Trabalho e Emprego. Os dados utilizados se referem ao ano de 2010*. Utilizaram-se também informações complementares do Conselho de registro profissional de enfermeiros (Conselho Regional de Enfermagem de Minas Gerais) de 2010 e da Pesquisa de Assistência Médico-Sanitária do Instituto Brasileiro de Geografia e Estatística (IBGE) de 2009.

Os dados do CNES e da RAIS foram escolhidos alternativamente aos dados do Conselho Regional de Enfermagem, que são geralmente consultados para dimensionar o quantitativo de profissionais habilitados ao exercício, não discriminando os que atuam ou não diretamente em serviços de saúde, ou mesmo os que não estão trabalhando na própria profissão. O CNES é a principal fonte para o dimensionamento do número de profissionais prestando assistência em saúde, além de fornecer o número de vínculos de trabalho na totalidade dos estabelecimentos de saúde. Por outro lado, a RAIS é o único registro que permite uma análise da profissão no total da economia, sendo considerado o melhor retrato disponível no Brasil sobre o mercado de trabalho formal.

A combinação foi utilizada para minimizar as limitações do uso isolado de cada banco de dados, a saber, a restrição do CNES aos estabelecimentos de saúde, não sendo possível contar os profissionais trabalhando na profissão em outros

* Os dados do INEP foram referentes ao volume de vagas, ingressos e egressos ao longo do ano; os dados do CNES, dos postos de trabalho ativos ao longo do mês de dezembro; e os da RAIS, aos vínculos ativos em 31 de dezembro de 2010. 
setores da economia; e a restrição da RAIS quanto aos dados referentes ao mercado formal e número de empregos, uma vez que os dados são coletados por vínculo de emprego e não por empregado. Em relação aos dados sobre os cursos de enfermagem, destacamos o limite quanto àqueles cursos que tiveram autorização para abertura a partir do Conselho Estadual de Educação e, que, portanto, não constavam na base de dados do INEP/MEC por ocasião da contagem do Censo.

Os dados foram tratados pela estatística descritiva. As informações obtidas foram inseridas em um banco de dados e a análise foi desenvolvida com o auxílio do programa Statistical Package for the Social Sciences (SPSS) versão 19.0. Os resultados estão apresentados em três categorias: A formação do enfermeiro em Minas Gerais; O mercado de trabalho do enfermeiro em Minas Gerais e Relações entre formação e mercado de trabalho.

\section{RESULTADOS}

\section{A formação do enfermeiro no Estado de Minas Gerais}

Os dados do Censo da Educação Superior revelam a existência de 122 cursos de Graduação em Enfermagem no Estado de Minas Gerais em 2010, sendo a maioria ofertada por instituições privadas. No cômputo geral, estes cursos ofereceram 12.574 vagas ao longo do ano, o equivalente a 0,64 por 1.000 habitantes em Minas Gerais. Destas vagas, 48,2\% foram ocupadas por 6.056 ingressos. Também em 2010, o Estado formou 6.429 enfermeiros, o correspondente a 0,33 por 1.000 habitantes.
Quando analisadas as diferentes regiões do Estado de Minas Gerais, os cursos estão concentrados majoritariamente nas macrorregiões Centro, com 31 cursos, Sul com 22 cursos, Oeste com 11 cursos e Sudeste com 10 cursos. A região do Jequitinhonha é a que possuía o menor número, apenas um curso.

Observamos uma maior taxa de aproveitamento das vagas na região Jequitinhonha que apresenta a melhor relação entre o número de ingressantes e vagas oferecidas nos cursos de Graduação em Enfermagem. Destaca-se, no entanto, que a região possui apenas 30 vagas, das quais 29 foram ocupadas. Por outro lado, as regiões Noroeste e Sudeste demonstram as menores taxas de ocupação das vagas, 25,8\% e 29\%, respectivamente.

A Figura 1 demonstra a expansão da formação de enfermeiros no Estado. Entre as décadas de 1990 e 2000, nota-se que o número de vagas, inscritos no vestibular, ingressantes e concluintes variavam sincronicamente, não apontando qualquer desregulação nessa relação. Após 1999, a evolução dos indicadores começa a se diferenciar, especialmente o de procura pelo curso, identificada pelo número de inscritos no vestibular. De fato, a procura começa a crescer bem antes disso, a partir de 1995, e alcança seu ápice 10 anos depois, em 2005, quando passa a declinar.

A ampliação do número de vagas acontece a partir de 2001 e, em função disso, o número de ingressos também aumenta, o que vai se refletir no número de concluintes entre 2005 e 2008. O ponto preocupante é a partir de 2004, quando o número de vagas ofertadas passa a ser maior que o número

Tabela 1 - Números do ensino superior de enfermagem em Minas Gerais por Macrorregião de Saúde - 2010

\begin{tabular}{|c|c|c|c|c|c|c|c|}
\hline Macrorregião de saúde & $\mathrm{N}^{0}$ de cursos & $\mathrm{N}^{\circ}$ de vagas & $\begin{array}{c}\mathrm{N}^{\circ} \mathrm{de} \\
\text { ingressos }\end{array}$ & $\begin{array}{l}\text { \% vagas } \\
\text { ocupadas }\end{array}$ & $\begin{array}{c}\mathrm{N}^{0} \text { de } \\
\text { concluintes }\end{array}$ & $\begin{array}{c}\text { Vagas } \\
\text { por hab.* }\end{array}$ & $\begin{array}{c}\text { Concluintes } \\
\text { por hab.* }\end{array}$ \\
\hline Centro & 31 & 4.096 & 2.349 & 57,3 & 2.082 & 0,67 & 0,34 \\
\hline Leste & 7 & 670 & 340 & 50,7 & 714 & 0,46 & 0,49 \\
\hline Triângulo do Sul & 4 & 345 & 241 & 69,9 & 266 & 0,49 & 0,38 \\
\hline Jequitinhonha & 1 & 30 & 29 & 96,7 & 26 & 0,11 & 0,09 \\
\hline Leste do Sul & 4 & 400 & 153 & 38,3 & 152 & 0,61 & 0,23 \\
\hline Sudeste & 10 & 1.460 & 424 & 29,0 & 364 & 0,93 & 0,23 \\
\hline Sul & 22 & 1.675 & 667 & 39,8 & 765 & 0,64 & 0,29 \\
\hline Centro Sul & 5 & 260 & 194 & 74,6 & 186 & 0,35 & 0,25 \\
\hline Nordeste & 5 & 600 & 130 & 21,7 & 102 & 0,67 & 0,11 \\
\hline Oeste & 11 & 760 & 370 & 48,7 & 463 & 0,64 & 0,39 \\
\hline Norte & 8 & 868 & 522 & 60,1 & 473 & 0,55 & 0,30 \\
\hline Noroeste & 6 & 620 & 160 & 25,8 & 346 & 0,95 & 0,53 \\
\hline Triângulo do Norte & 8 & 790 & 477 & 60,4 & 490 & 0,67 & 0,42 \\
\hline Minas Gerais & 122 & 12.574 & 6.056 & 48,2 & 6.429 & 0,64 & 0,33 \\
\hline
\end{tabular}

Fonte: Censo da Educação Superior do INEP e do Censo Demográfico do IBGE.

*Por 1.000 habitantes. 


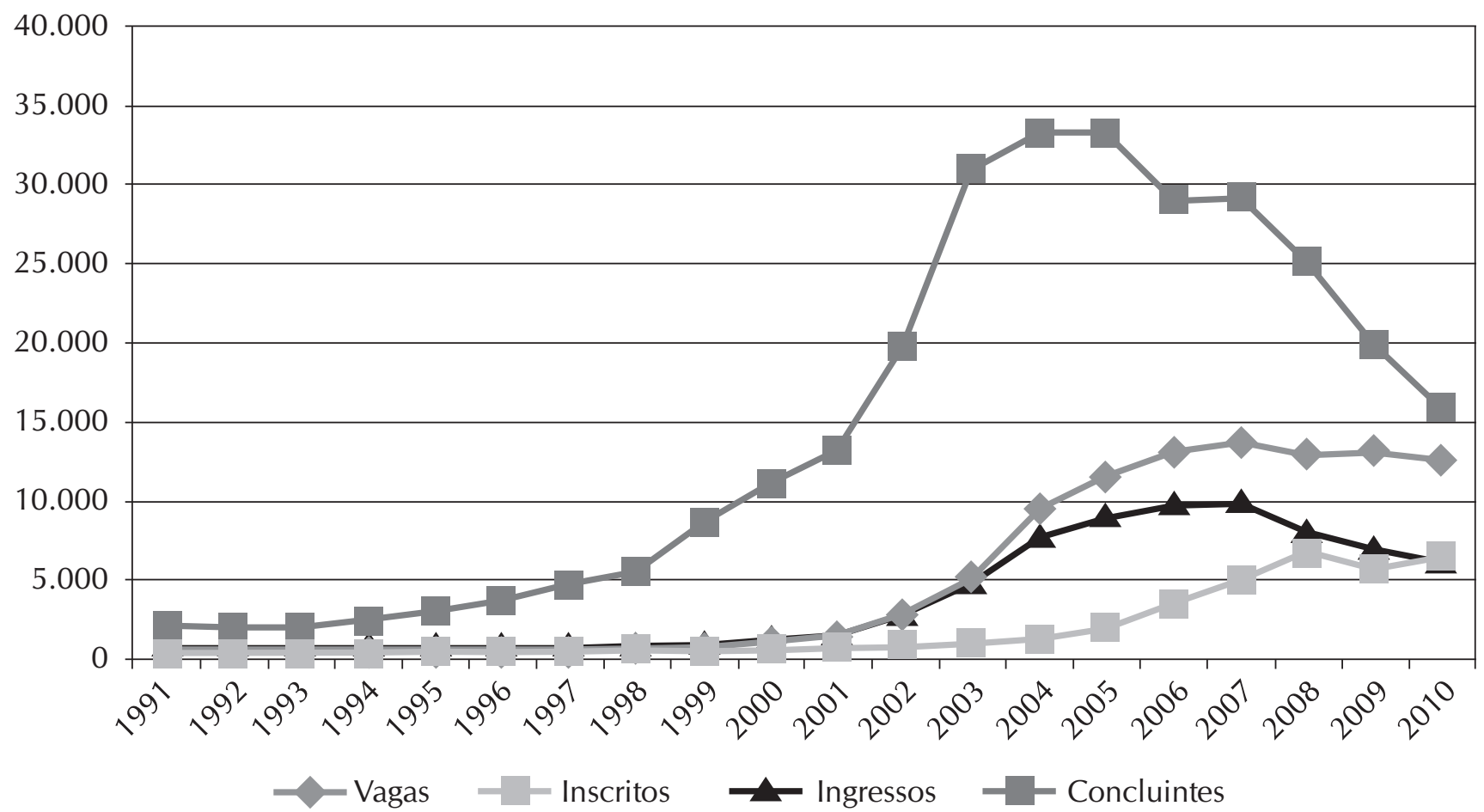

Figura 1 - Evolução do número de vagas, inscritos no vestibular, ingressos e concluintes dos cursos de Enfermagem - Minas Gerais, 1991 a 2009.

Fonte: Censo da Educação Superior do INEP.

de ingressos, indicando uma taxa de desocupação daquelas. De 2007 para 2008, observa-se uma discreta diminuição no número de vagas ofertadas, mostrando uma reação imediata do sistema formador à diminuição do interesse pelo curso e ao aumento da desistência de matriculados. Neste último aspecto, os dados revelam que, em todo o período da expansão, o número de concluintes sempre foi menor que o de ingressantes indicando a taxa de evasão nos cursos de Graduação em Enfermagem.

Mercado de trabalho do enfermeiro em Minas Gerais: estoque de enfermeiros, vínculos em estabelecimentos de saúde e empregos formais

Os achados do estudo demonstram que o número de profissionais registrados no Conselho, ativos em 2010, é de 29.496 enfermeiros. Destes, 13.366 constam no CNES, como ocupados em estabelecimentos de saúde. Assim, em torno de $45 \%$ do total de inscritos ativos no Conselho possuíam vínculo na assistência à saúde como enfermeiros, no núcleo do setor. A outra parcela corresponde a pessoas trabalhando na profissão em outros setores da economia, ou mesmo na saúde, mas em funções administrativas ou de ensino e pesquisa. Infere-se que alguns profissionais podem estar atuando como Técnicos ou Auxiliares de Enfermagem. Ainda podem ser considerados aqueles que têm registro, mas estão trabalhando em outras funções, sem relação direta com a Enfermagem e a área da saúde, profissionais desocupados e aqueles sem qualquer posição no mercado de trabalho.

A comparação entre os números de postos ou vínculos de trabalho de enfermeiros em estabelecimentos de saúde no CNES e na AMS, respectivamente de 15.570 e 15.473, destaca que, a despeito das diferenças e limites entre as bases, confirma-se uma estimativa em torno de 15,5 mil postos de trabalho de enfermeiros, na assistência direta à saúde no Estado de Minas Gerais, em 2010. Se considerarmos que a Enfermagem é uma categoria extremamente formalizada, o número de 16.377 empregos formais, disponibilizados pela RAIS, corrobora tal estimativa e permite inferir que a grande maioria dos ocupados na área se encontra na assistência.

O número de profissionais Full Time Equivalent (FTE), de 13.256, equivale ao de enfermeiros ajustados por 40 horas integrais de trabalho. $\mathrm{O}$ valor do FTE, próximo ao de profissionais registrados no CNES, demonstra que os vínculos de trabalho de enfermeiros em Minas Gerais são, em média, muito próximos de 40 horas semanais. Esse achado corrobora a distribuição da carga horária semanal dos enfermeiros ativos e registrados na RAIS, na qual consta que 34\% dos empregos da categoria enfermeiro estão registrados com carga horária semanal acima de 40 horas, 35\% com carga horária entre 31 e 40 horas; $23 \%$ com carga horária entre 21 e 30 horas e $8 \%$ com carga horária até 20 horas.

Quando analisamos os enfermeiros cadastrados em estabelecimentos de saúde, observa-se que o maior número de vínculos está situado nas regiões mais desenvolvidas do Estado 
Tabela 2 - Razão entre o número de Enfermeiros em estabelecimentos de saúde e o número de empregos formais de Enfermeiros no total da economia - Minas Gerais, 2010.

\begin{tabular}{|c|c|c|c|c|c|}
\hline Macrorregião & $\mathrm{N}^{0}$ de profissionais ${ }^{(a)}$ & $\begin{array}{c}\text { Razão profissionais/ } \\
1.000 \text { hab. }\end{array}$ & $\begin{array}{c}N^{0} \text { de empregos } \\
\text { formais }^{(b)}\end{array}$ & $\begin{array}{c}\text { Razão empregos/ } \\
\text { profissionais }\end{array}$ & $\begin{array}{c}\text { Proporção trabalho } \\
\text { protegido }^{(c)}\end{array}$ \\
\hline Centro & 4.920 & 0,81 & 6.810 & 1,38 & 30,8 \\
\hline Leste & 832 & 0,57 & 954 & 1,15 & 63,6 \\
\hline Triângulo do Sul & 558 & 0,80 & 679 & 1,22 & 26,6 \\
\hline Jequitinhonha & 191 & 0,51 & 125 & 0,65 & 54,1 \\
\hline Leste do Sul & 407 & 0,61 & 390 & 0,96 & 77,1 \\
\hline Sudeste & 1.128 & 0,72 & 1.298 & 1,15 & 49,3 \\
\hline Sul & 1.669 & 0,64 & 2.240 & 1,34 & 40,8 \\
\hline Centro Sul & 449 & 0,62 & 469 & 1,04 & 53,8 \\
\hline Nordeste & 560 & 0,69 & 345 & 0,62 & 57,8 \\
\hline Oeste & 671 & 0,56 & 842 & 1,25 & 57,6 \\
\hline Noroeste & 375 & 0,57 & 338 & 0,90 & 51,8 \\
\hline Norte & 1.002 & 0,64 & 877 & 0,88 & 74,6 \\
\hline Triângulo do Norte & 672 & 0,57 & 1.002 & 1,49 & 51,0 \\
\hline Minas Gerais & 13.366 & 0,68 & 16.377 & 1,23 & 44,8 \\
\hline
\end{tabular}

Fonte: Cadastro Nacional de Estabelecimentos de Saúde e da Relação Anual de Informações Sociais.

(a) Número de profissionais em estabelecimentos de saúde - CNES;

(b) Número de vínculos formais de emprego, ativos em 31/12 - RAIS;

(c) Proporção de vínculos empregatícios estatutários ou regidos pela CLT de enfermeiros em estabelecimentos de saúde.

(Centro, Sul e Sudeste), conforme demonstrado na Tabela 2.

Tomando o número de enfermeiros em estabelecimentos de saúde como uma aproximação da oferta de profissionais no cálculo da razão entre empregos formais e enfermeiros por Macrorregião, nota-se que as regiões Triângulo do Norte, Centro, Sul, Triângulo do Sul, Sudeste, Leste, Centro Sul e Oeste apresentam um número de empregos superior ao de profissionais registrados no CNES. No Triângulo Norte, que tem a maior razão, o número é de 149 empregos formais para cada 100 profissionais que estão registrados no CNES. Ao passo que no Nordeste, são 62 empregos formais para cada 100 enfermeiros. Razão menor que 1,0 pode significar um escassez na oferta de empregos, bem como a presença da enfermagem no mercado informal. Por outro lado, razões maiores que 1,0 , revelam uma importante presença de oferta de empregos para enfermagem em outros setores, que não em estabelecimentos de saúde, bem como a ocupação de mais de um emprego por enfermeiro.

Se comparadas as regiões do Estado, os dados apresentados revelam menor proporção de trabalho protegido (vínculos empregatícios estatutários ou regidos pela CLT em estabelecimentos de saúde) nas regiões menos desenvolvidas do Estado, com especial atenção às regiões Leste do Sul, Norte e Leste que registraram, respectivamente, $22,9 \%, 25,4 \%$ e $36,4 \%$ de trabalho protegido em relação ao total.

\section{Relação entre formação e mercado de trabalho}

A Figura 3 demonstra a relação entre o número de vagas em cursos de Graduação em Enfermagem e o número de postos de trabalho cadastrados para enfermeiros nas diferentes regiões do Estado, em 2010*. Trata-se de um quociente que representa a distribuição da oferta efetiva do sistema formador e de mercado de trabalho entre as macrorregiões do Estado, no que se refere à Enfermagem. Evidenciamos um desequilíbrio entre o número de profissionais que potencialmente são formados e sua possível absorção no mercado de trabalho, mesmo que o quantitativo de profissionais não seja suficiente para ampliar a razão entre o número de enfermeiros e o número de habitantes, o que significaria potencialidade para qualificar o atendimento às necessidades de saúde da população.

Das 13 macrorregiões de saúde do Estado, 7 apresentaram valores do quociente entre vagas e empregos abaixo de 1, o que significa uma escassez de vagas em relação aos empregos, ou de outra forma, uma concentração de empregos em relação ao número de vagas, comparativamente às demais

* Quociente entre a razão de vagas na macrorregião e de vagas no Estado, por um lado, e a razão do número de empregos na macrorregião e de empregos no Estado, por outro lado. 


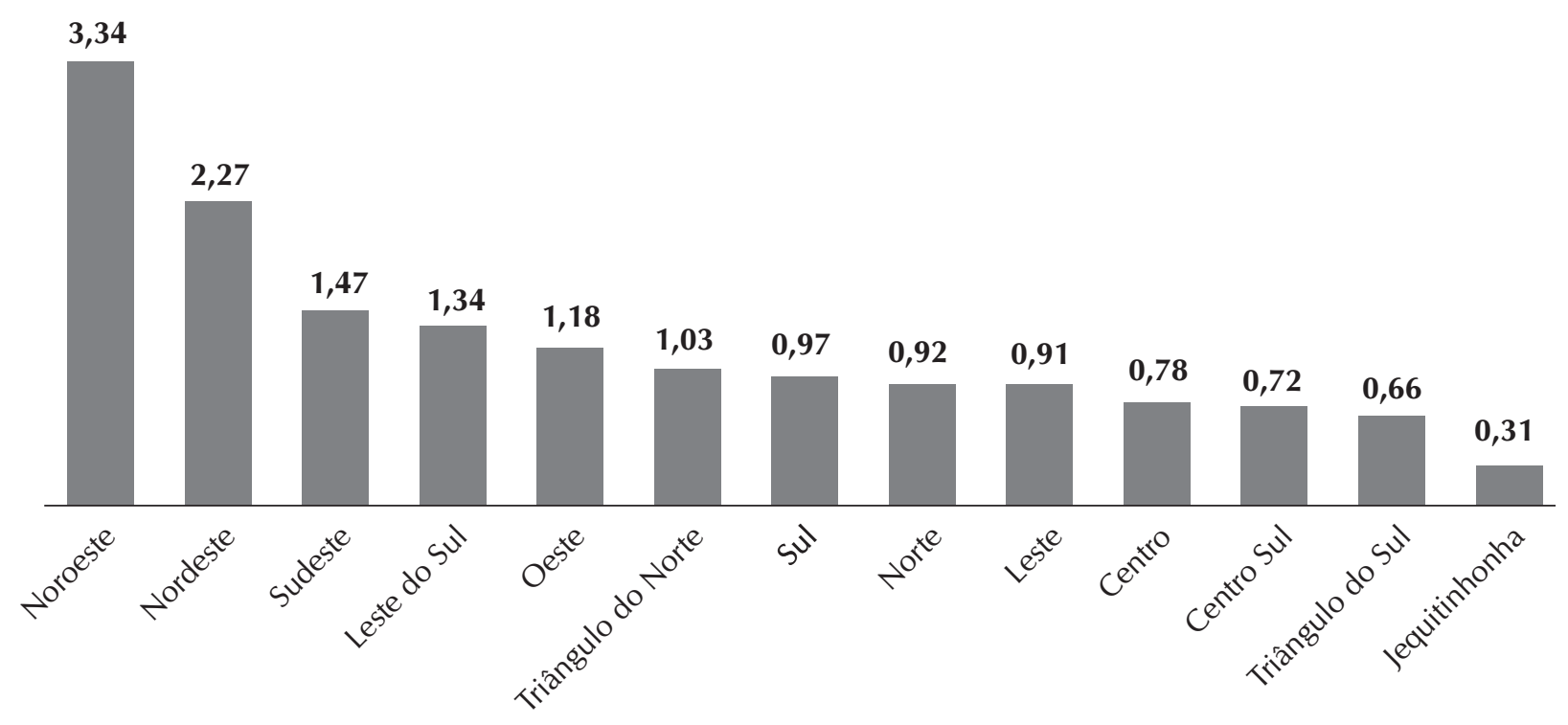

Figura 03 - Quociente entre o número de vagas em cursos de enfermagem e vínculos formais de emprego por macrorregião de saúde - Minas Gerais, 2010.

Fonte: Censo da Educação Superior do INEP e da Relação Anual de Informações Sociais.

macrorregiões. Por outro lado, 6 registraram valores superiores. A região Noroeste tem o maior quociente, 3,34, isto é, possui um número de vagas de enfermagem 224\% superior à oferta de empregos formais de enfermeiros. Para as regiões Triângulo Norte e Sul, verificaram-se valores muito próximos do que seria considerada uma igualdade na distribuição de vagas e empregos, em relação às demais regiões de Minas Gerais.

\section{DISCUSSÃO}

Os achados corroboram outros estudos que demonstram trajetórias de expansão na formação de enfermeiros e outros profissionais de saúde no país ${ }^{(2,8-10)}$. Há dois momentos de destaque para o fenômeno: entre 1970 e 1985 e entre 1995 e 2010. Em ambos, observamos uma tendência expansionista de cursos e instituições, reflexo de políticas governamentais de ampliação de vagas e acesso ao ensino superior no Brasil.

No primeiro período, por impulso da Reforma Universitária de 1968, houve ampliação em termos quantitativos do grau superior de escolarização, da população de classe média, principalmente nas áreas técnicas e tecnológicas, de modo a produzir o "capital humano" necessário para impulsionar o desenvolvimento econômico do país ${ }^{(11)}$; e no segundo, reflexo da Lei de Diretrizes e Bases da Educação Nacional (LDB) que forneceu autonomia pedagógica, administrativa e de gestão financeira para as instituições de ensino, ocorreu um aumento do número de instituições de ensino superior ${ }^{(9)}$. Somam-se também, no período recente, os investimentos para inclusão de camadas mais pobres no ensino superior, como o Programa Universidade para Todos (ProUni) que, ao mesmo tempo, surge como alternativa compensatória para as instituições privadas frente ao excessivo número de vagas criadas no inicio dos anos 2000, e que muitas vezes tornaram-se ociosas ${ }^{(11)}$.

No caso particular da Enfermagem, na década de 1970, o crescimento do número de cursos e escolas de enfermagem foi assumido como uma solução para o déficit de enfermeiros no país. Contudo, não houve crescimento correspondente na procura pelos cursos de graduação e, consequentemente, não houve alterações significativas na composição da força de trabalho da Enfermagem e na ocupação do mercado de trabalho ${ }^{(8)}$.

No período recente, a partir de 1995, o que se observa é o movimento inverso, com aumento da procura pelos cursos de Graduação em Enfermagem e, posteriormente, a ampliação de vagas para responder a essa demanda. O aumento da procura pode estar relacionado à implementação do Sistema Único de Saúde (SUS), com expansão de postos de trabalho; e à implantação do Programa Saúde da Família (PSF), que se tornou uma perspectiva atraente no mercado de trabalho dos enfermeiros $^{(8)}$. A esse respeito, a empregabilidade da categoria atingiu no pais índices de 92,4\% de absorção em 1999 quando grande parte de outras profissionais do setor saúde enfrentava crises de desemprego ${ }^{(12)}$.

Contudo, com o crescimento exponencial e desordenado dos cursos de graduação, em especial a partir de 2002, retornamos a um momento de diminuição da procura, reflexo do novo contexto da empregabilidade dos enfermeiros nas instituições de saúde e da representação sobre os empregos para a categoria.

No cenário atual, existem diversos fatores advindos do mercado de trabalho que parecem determinar a trajetória dos cursos de graduação. De um lado, o aparente excedente de 
pessoal demonstrado pela diferença entre os números de profissionais formados, com registro no conselho de classe, e a capacidade de absorção nos vínculos existentes nos estabelecimentos de saúde e nos empregos formais, indicando que há tendência de desemprego e informalidade nos vínculos de trabalho do enfermeiro, bem como abandono da profissão. Somam-se a isso as transformações dos vínculos, das condições de trabalho e da remuneração do pessoal de enfermagem que parecem produzir um efeito negativo na procura pelo curso, ainda que permaneça a necessidade de contratação de enfermeiros para fazer frente às principais modalidades de organização dos serviços de saúde. Em especial, destacamos a desregulamentação da carga horária e de pisos salariais para a categoria que leva os profissionais a "aceitar" as condições impostas pelo mercado.

Destaca-se que o excedente de enfermeiros é aparente, pois quando analisamos a distribuição do número de profissionais pela população, em todas as regiões do Estado, verificamos índices abaixo do preconizado por organismos internacionais e encontrados em outros países do mundo, bem como no próprio país ${ }^{(8)}$.

Assim, o possível "desemprego" que influencia a procura pelos cursos de Graduação em Enfermagem está associado à não expansão dos postos de trabalho. Com isso, evidencia-se a urgência da discussão sobre a regulação da formação profissional, assim como a necessidade de expansão de postos de trabalho que sejam mais adequados às necessidades de saúde da população.

Os achados do estudo revelam o ciclo vicioso de reprodução de desigualdades na relação entre a oferta e a demanda por profissionais de enfermagem com variações significativas nas diferentes regiões do Estado de Minas Gerais. Considerando a divisão territorial do Estado a partir do Plano de Regionalização da Saúde, Minas Gerais apresenta 13 macrorregiões sendo o Vale do Jequitinhonha e Mucuri e o Norte de Minas regiões pobres, caracterizadas pela deficiência na infraestrutura social, baixa incorporação tecnológica e poucos investimentos econômicos. As regiões Centro, Centro-Oeste e Sul caracterizam-se pela alta industrialização. Já as regiões do Triangulo Norte e Sul possuem uma economia baseada na agropecuária com grandes investimentos agroindustriais.

A concentração de investimentos e de infraestrutura nas regiões mais desenvolvidas do Estado determina a concentração de cursos nestas regiões o que, por sua vez, gera maior oferta de profissionais. Esse aspecto foi revelado no estudo na análise da relação positiva entre o número de cursos existentes, número de vagas nos cursos, número de vínculos em estabelecimentos de saúde e número de empregos formais de enfermeiros nestas regiões e suscita a discussão quanto à desiguladade na distribuição dos profissionais no Estado e à reprodução das condições que agravam as iniquidades como o acesso a assistência à saúde, incluindo-se a disponibilidade dos diferentes profissionais.

Outros estudos sobre a formação de profissionais e a distribuição de recursos humanos de saúde também revelam essa desigualdade identificando que nas regiões Sul e Sudeste do país há melhores índices na relação egresso dos cursos de graduação da área de saúde/habitante enquanto as regiões Norte e Nordeste possuem os piores índices ${ }^{(2,13)}$. De forma similar, a maior parte das escolas médicas e dos programas de residência médica, concentra-se nas áreas metropolitanas, em especial da região Sudeste do país, mais especificamente as regiões metropolitanas de São Paulo e Rio de Janeiro ${ }^{(13)}$.

Reforça-se que acúmulo de expansões de oferta e demanda, determinadas por lógicas mercadológicas, do setor educacional ou do setor saúde, para fazer frente aos interesses individuais de um ou outro setor, produz efeitos negativos tanto na abertura de vagas para a formação, como na criação de postos de trabalho com acentuados desequilíbrios regionais. Tal cenário, aponta para a urgente articulação entre a formação profissional e a organização do sistema de saúde, mediatizado pelo quadro sanitário, e influenciado pelas oscilações de um mercado em transformação ${ }^{(14)}$.

\section{CONSIDERAÇÕES FINAIS}

Constata-se a expansão dos cursos de graduação de enfermagem, dos ingressantes e dos egressos no Estado de Minas Gerais a partir de 2000 e indícios de diminuição a partir de 2007, que parecem se relacionar às mudanças no mercado de trabalho dos enfermeiros.

O aumento da empregabilidade proporcionado pela implantação do SUS elevou a procura pelo curso de enfermagem, repercutindo na ampliação de vagas, cursos, bem como de instituições de ensino superior. Entretanto, com a expansão exponencial e desordenada, delineia-se um novo contexto de empregabilidade, principalmente a partir de 2002, repercutindo em vagas ociosas nos cursso de graduação. Pode-se inferir que nos próximos anos haverá queda acentuada nos concluintes e redução da oferta de cursos, devido à diminuição dos ingressantes e ao índice de evasão nos cursos de enfermagem observados a partir de 2007.

Os resultados permitem afirmar que há tendência de desemprego para a categoria de enfermeiros. O desemprego pode estar associado a não expansão dos postos de trabalho, a ocupação de mais de um posto por um mesmo profissional e o desequilíbrio na oferta e demanda dos profissionais, relacionado à reprodução das desigualdades socioeconômicas nas regiões do Estado.

Há também outros elementos que precisam ser considerados na ocupação de postos no mercado informal, que não foi objeto desta análise frente ao limite dos bancos de dados fontes da pesquisa, mas que estimulam a continuidade de novos estudos.

Destaca-se que o excedente de enfermeiros no mercado de trabalho é aparente, pois o índice de número de profissionais por habitantes no Estado ainda é abaixo do preconizado para atender as necessidades de saúde da população. A lógica mercadológica tem determinado a expansão dos cursos e sua concentração, sendo urgente a discussão sobre a regulação da formação profissional e da necessidade de expansão dos postos de trabalho. 


\section{REFERÊNCIAS}

1. Ito EE, Peres AM, Takahashi RT, Leite MMJ. O ensino de enfermagem e as diretrizes curriculares nacionais: utopia x realidade. Rev Esc Enferm USP 2006;40(4):570-5.

2. Haddad AE, Morita MC, Pierantoni CR, Brenelli SL, Passarella T, Campos FE. Formação de profissionais de saúde no Brasil: uma análise no período de 1991 a 2008. Rev Saúde Pública 2010; 44(3):383-93.

3. Sena RR, Seixas CT, Silva KL. Practices in Community Health Toward Equity: contributions of Brazilian Nursing. ANS Adv Nurs Sci 2007;30(3):343-52.

4. Rigoli F, Rocha CF, Foster AA. Desafios críticos dos recursos humanos em saúde: uma visão regional. Rev Latinoam Enferm 2006;14(1):7-16.

5. Varella TC, Pierantoni CR. Mercado de trabalho: revendo conceitos e aproximando o campo da saúde. A década de 90 em destaque. Physis 2008;18(3):521-44.

6. Ramos LO. Desempenho recente do mercado de trabalho brasileiro: tendências, fatos estilizados e padrões espaciais. Brasil, o Estado de uma nação. Ministério do Planejamento, Orçamento e Gestão. Rio de Janeiro: Ipea; 2006.

7. Simão RCS. A contribuição das parcelas da renda para a desigualdade do rendimento domiciliar per capita em Minas gerais de acordo com os dados do Censo Demográfico 2000. Pesqui debate 2008; 19(1):33-44.

8. Varella TC, Pierantoni CR. A migração de enfermeiros: um problema de saúde pública. Rev Bras Saúde Mater Infant 2007;7(2):199-211.

9. Vieira ALS, Garcia ACP, Filho AA, Pierantoni CR, Ferraz $\mathrm{CP}$, Oliveira ES, et al. Tendências do sistema educativo no Brasil: Medicina, Enfermagem e Odontologia. In: Ministério da Saúde (Brasil). Observatório de Recursos Humanos em Saúde no Brasil: estudos e análises. Brasília: Ministério da Saúde; 2004.

10. Teixeira E, Vale EG, Fernandes JD, Sordi MRL. Trajetória e tendências dos cursos de Enfermagem no Brasil. Rev Bras Enferm 2006;59(4):479-87.

11. Carvalho CHA. Política de ensino superior e renúncia fiscal: da reforma universitária de 1968 ao PROUNI. Universidade de Campinas; 2008.

12. Vieira ALS. Empregabilidade dos enfermeiros no Brasil. Rev Enferm UERJ 2002;(6 Suppl):S65-74.

13. Ceccim RB, Pinto LF. A formação e especialização de profissionais de saúde e a necessidade política de enfrentar as desigualdades sociais e regionais. Rev Bras Educ Med 2007;31(3):266-277.

14. Pierantoni CR, França T, Varella TC. Evolução da oferta de profissionais médicos e enfermeiros no Brasil: disponibilidade do sistema educacional para a formação. In: Ministério da Saúde (Brasil), organizador. Observatório de recursos humanos em saúde no Brasil. Rio de janeiro: Editora Fiocruz; 2003, v. 1, p. 279-292. 